\title{
Navigated ultrasound-based image guidance during resection of gliomas: practical utility in intraoperative decision-making and outcomes
}

\author{
Prakash Shetty, MCh, ${ }^{1,2}$ Ujwal Yeole, MCh, ${ }^{1,2}$ Vikas Singh, $M C h,{ }^{1,2}$ and Aliasgar Moiyadi, $\mathrm{MCh}^{1,2}$ \\ ${ }^{1}$ Neurosurgical Services, Department of Surgical Oncology, Tata Memorial Centre, and ${ }^{2}$ Homi Bhabha National Institute, Mumbai, \\ India
}

\begin{abstract}
OBJECTIVE Intraoperative imaging is increasingly being used for resection control in diffuse gliomas, in which the extent of resection (EOR) is important. Intraoperative ultrasound (iUS) has emerged as a highly effective tool in this context. Navigated ultrasound (NUS) combines the benefits of real-time imaging with the benefits of navigation guidance. In this study, the authors investigated the use of NUS as an intraoperative adjunct for resection control in gliomas.
\end{abstract}

METHODS The authors retrospectively analyzed 210 glioma patients who underwent surgery using NUS at their center. The analysis included intraoperative decision-making, diagnostic accuracy, and operative outcomes, particularly EOR and related factors influencing this.

RESULTS US-defined gross-total resection (GTR) was achieved in $57.6 \%$ of patients. Intermediate resection control scans were evaluable in 115 instances. These prompted a change in the operative decision in $42.5 \%$ of cases (the majority being further resection of unanticipated residual tumor). Eventual MRI-defined GTR rates were similar (58.6\%), although the concordance between US and MRI was $81 \%$ (170/210 cases). There were 21 false positives and 19 false negatives with NUS, resulting in a sensitivity of $78 \%$, specificity of $83 \%$, positive predictive value of $77 \%$, and negative predictive value of $84 \%$. A large proportion of patients (13/19 patients, $68 \%$ ) with false-negative results eventually had near-total resections. Tumor resectability, delineation, enhancement pattern, eloquent location, and US image resolution significantly influenced the GTR rate, though only resectability and eloquent location were significant on multivariate analysis.

CONCLUSIONS NUS is a useful intraoperative adjunct for resection control in gliomas, detecting unanticipated tumor residues and positively influencing the course of the resection, eventually leading to higher resection rates. Nevertheless, resection is determined by the innate resectability of the tumor and its relationship to eloquent location, reinforcing the need to combine iUS with functional mapping techniques to optimize resections.

https://thejns.org/doi/abs/10.3171/2020.10.FOCUS20550

KEYWORDS diagnostic accuracy; glioma surgery; intraoperative ultrasound; navigated ultrasound; resection control

$\mathrm{D}$ IFFUSE gliomas remain a challenging group of tumors to address surgically. The extent of resection (EOR) has been proven to be an important prognostic factor, and various strategies to improve intraoperative assessment of resection have been developed. Intraoperative ultrasound (iUS) has emerged as a useful and cost-effective multifaceted imaging modality. ${ }^{1}$ Navigated ultrasound (NUS) is a powerful tool that combines the benefits of real-time 2D ultrasound (2D US) imaging with the ability to track the US probe, allowing for imageguided navigation. ${ }^{2}$ NUS can be used alone (direct NUS $[\mathrm{DNUS}])^{3}$ but is more often used in combination with pre- operative MRI (image fusion mode). In our experience, we have found NUS to be very useful for performing biopsies as well as resecting glioblastomas and nonenhancing gliomas. ${ }^{4-6}$ The major purpose of NUS (as with most image guidance and intraoperative adjuncts) is lesion localization (which is used for biopsy targeting as well as for lesion identification and trajectory planning during the resection of some tumors, especially well-delineated masses like metastases and some gliomas) and resection control (primarily for gliomas, where NUS is used for performing serial resection scans to assess the resection in real time and plan the further progress of the surgical procedure). NUS

ABBREVIATIONS ALA = aminolevulinic acid; DNUS = direct NUS; EOR = extent of resection; GTR = gross-total resection; HGG = high-grade glioma; IGS = image guidance system; iUS = intraoperative ultrasound; LGG = low-grade glioma; NPV = negative predictive value; NTR = near-total resection; NUS = navigated ultrasound; PPV = positive predictive value; STR = subtotal resection; $2 \mathrm{D}$ US $=2 \mathrm{D}$ ultrasound.

SUBMITTED June 13, 2020. ACCEPTED October 8, 2020.

INCLUDE WHEN CITING DOI: 10.3171/2020.10.FOCUS20550. 
is particularly suited for resection control, offering quick and repeated updates of the operative field/cavity without significantly interfering with the surgical workflow. Intermediate US scans can be serially performed multiple times and, when navigated, offer the possibility of spatial and temporal correlation across serial scans, thereby improving the reliability of the interpretation. In the present paper, we report our analysis of NUS used in operations performed in a homogenous cohort of diffuse glioma patients and our objective evaluation of the influence of the use of NUS on the progress of the resections as well as the eventual patient outcomes.

\section{Methods}

This was a retrospective descriptive cohort study. The study was approved by the institutional ethics committee (IEC no. 900632). Informed consent was not required as there was no direct contact with any of the subjects. We used a NUS-enabled image guidance system (IGS) (Sonowand AS) from 2010 through 2019. Representative images of the IGS and the probes are provided in Supplementary Fig. 1. The system, which has been described previously, ${ }^{5,7}$ essentially combines navigation technology with a high-end dedicated cranial insonation probe capable of generating 2D as well as 3D images. The cranial probe is precalibrated and registered to the navigation system (the probe as well as the acquired images are in the same frame of reference, and hence accuracy is enhanced). The system can rapidly (within 30-40 seconds) acquire a series of 2D images (about 200-300), which are computed automatically into a 3D volume that can then be displayed on the navigation system in either the traditional ACS (axial, coronal, sagittal) planes or a more user-friendly and intuitive "dual-anyplane" mode. The US data can also be superimposed on preoperative MR images (when available) to provide a better orientation of the cross-sectional anatomy. This data set can then be repeatedly updated (as and when necessary) during the course of the surgery. The IGS can function as a stand-alone 2D US, navigation system, as well as a 3D NUS device. NUS can be used alone (DNUS, where only NUS images are used without preoperative MRI) or with multimodal image fusion (where both preoperative MR images and intraoperative navigated and coregistered US images are used). The probes used included the 8FPA (3- to $8-\mathrm{MHz}$ phased array, $25 \times 17-\mathrm{mm}$ footprint), 10FPA-MC (5- to $10-\mathrm{MHz}$ phased array, $15 \times 13-\mathrm{mm}$ footprint-burr hole probe), and 12FLA (6- to $12-\mathrm{MHz}$ linear array, $32 \times 11-$ $\mathrm{mm}$ footprint). All consecutive cases where this system was used are recorded in a prospective database, which was queried to retrieve all the data. Some of the data related to specific patient subgroups have been published earlier. ${ }^{3,5-7}$ For the present analyses, the overall pattern of use and influence of NUS on the intraoperative decisionmaking and final resection in gliomas were analyzed. The database captures details including the intent of surgery, tumor characteristics based on preoperative MRI (delineation, eloquence, enhancement pattern, tumor resectability), US mode used, probe, and image resolution, as well as intraoperative imaging findings. For all of the cases in which US was used in some form, the database recorded the purpose of the use of US (for tumor localization or resection control) as well as the number of intermediate NUS scans performed, including the surgeon's impression (residue anticipated or not) and the US findings at each stage. The final control US finding is also recorded. All of these data are prospectively documented by the operating surgeon immediately after the surgery. Further clinical and perioperative details were retrieved by querying the hospital information system and the PACS for radiology reports. To record the concordance between the final control US scan and postoperative MRI and calculate the diagnostic accuracy of the US in predicting residue, only those cases for which a postoperative MRI was available were included. Gross-total resection (GTR) was defined as the absence of any contrast enhancement or T2/FLAIR abnormality (in nonenhancing tumors) as reported by our radiologists routinely. Volumetric analysis of residue is not routinely performed. Nonglial cases were excluded to maintain a homogeneous cohort of cases. The use of any additional intraoperative adjuncts (fluorescence, neurophysiological mapping, and awake mapping) was also recorded.

\section{Statistical Analysis}

Descriptive analysis was performed for all variables. Categorical data were reported as percentages. Univariate analysis using the chi-square (Pearson) test was carried out to identify predictors of GTR. All potential predictors were analyzed in a multivariate logistic regression model, using GTR as the outcome variable. Backward stepwise deletion based on the Wald test was applied. The overall performance of the internally validated model was assessed using Nagelkerke $\mathrm{R}^{2}$ (the higher the Nagelkerke $\mathrm{R}^{2}$, the greater the strength of the model). Finally, the HosmerLemeshow goodness-of-fit statistic was computed as a quantitative measure of accuracy. The model with the best fit was selected and reported. For all tests, $p$ values were two-sided, and a $\mathrm{p}$ value $<0.05$ was considered statistically significant. The diagnostic accuracy of the final resection control US scan (reported as "no residual tumor") was assessed using the radiologist-reported postoperative resection control MR image (reported as "no residual tumor") as the gold standard and employing $2 \times 2$ tables to calculate various parameters (sensitivity, specificity, and predictive values). All statistical analysis was done by using IBM SPSS (version 25, IBM Corp.).

\section{Results}

Figure 1 shows the outline of the study design. In total, 450 cases of various brain tumors were operated using image guidance during this period. Lobar gliomas accounted for a large proportion of the overall cases, with a large majority of them being planned for radical debulking surgery with NUS used for resection control. Table 1 describes the clinical, demographic, and tumor-related characteristics of the entire cohort of 450 cases.

Overall, US was used as part of the IGS plan in the vast majority of cases $(375 / 450,83.5 \%)$, primarily as NUS (368 cases) and occasionally as 2D US (7 cases). Intraopera- 


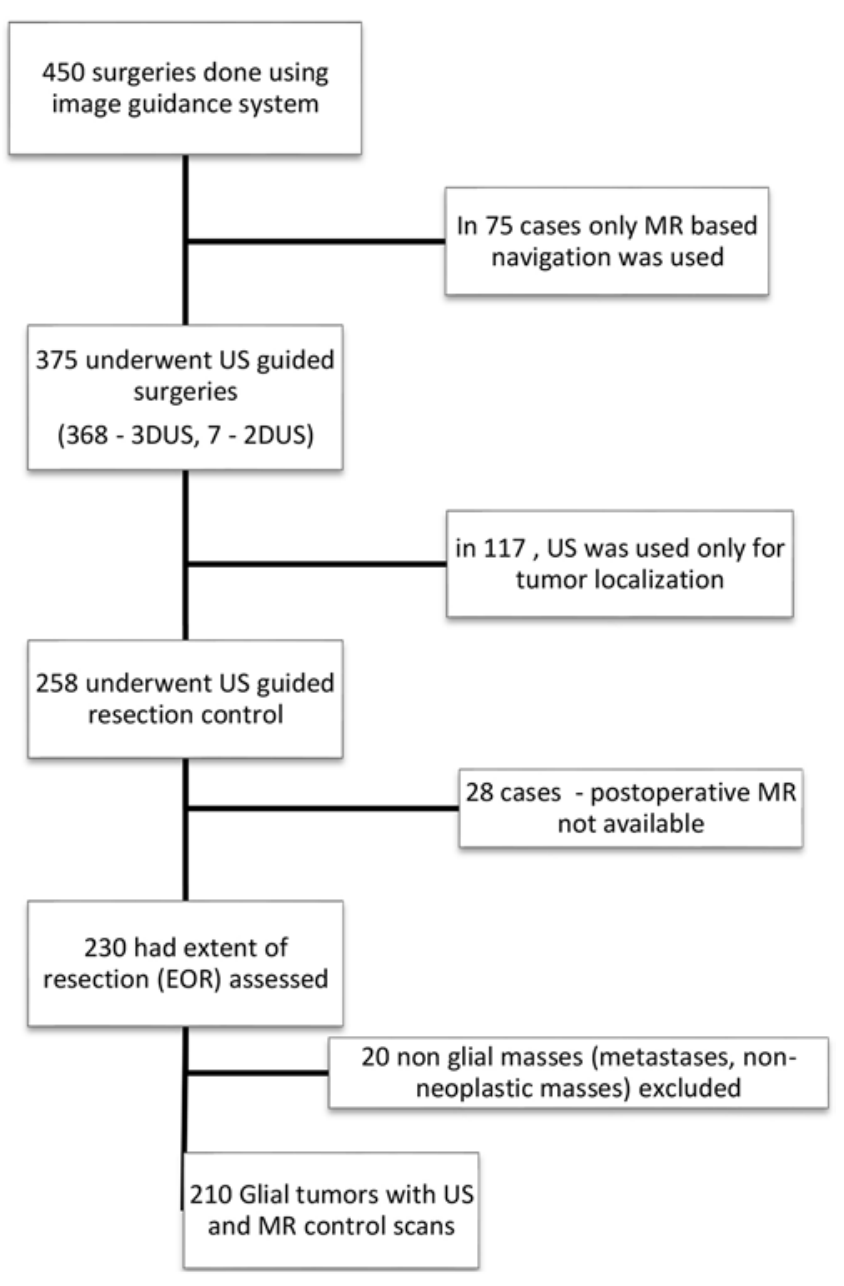

FIG. 1. Flow diagram showing the study cohort selection and analysis.

tive US was used for resection control in 258 of the 375 cases (NUS was used in 256 cases; DNUS in 155 cases; iUS in combination with MRI, i.e., image fusion, in 101 cases; and 2D US in 2 cases). In the remaining 117 cases, iUS was used only as a localizing tool (either for a biopsy or to localize a lesion for resection, but not as a resection control tool). After excluding nonglial tumors (like metastases) as well as some gliomas where postoperative MRI could not be performed, eventually NUS was used for resection control in 210 gliomas (130 DNUS, 80 image fusion). Table 1 shows the comparative demographic features of this group. Among the gliomas, high-grade gliomas (HGGs) constituted $83 \%$ of the total (10 grade 1, 26 grade 2, 86 grade 3, and 88 grade 4 ). Forty-five percent of these gliomas did not have any contrast enhancement. Figures 2-4 illustrate typical examples of gliomas resected using NUS (both DNUS and image fusion NUS). Intraoperative US images were reported of good or fair quality in $56 \%(116 / 210)$ and $41 \%(88 / 210)$ of cases, respectively, with only 6 IUS images $(3 \%)$ being recorded as poor.

\section{Decision-Making With NUS- and US-Defined GTR}

No intermediate scans were performed in 97 glioma cases in which the surgeon only performed a final control
TABLE 1. Profile of cases in the overall and glioma cohorts

\begin{tabular}{|c|c|c|}
\hline & \multicolumn{2}{|c|}{ Tumor Cohort/Op } \\
\hline & $\begin{array}{l}\text { Overall//mage } \\
\text { Guided }(n=450)\end{array}$ & $\begin{array}{l}\text { Gliomas/US-Based } \\
\text { Resection }(n=210)\end{array}$ \\
\hline \multicolumn{3}{|l|}{ Demographic data } \\
\hline Age, yrs, mean (range) & $42.3(4-81)$ & $40.8(6-77)$ \\
\hline Male sex & $292(65 \%)$ & $147(70 \%)$ \\
\hline \multicolumn{3}{|l|}{ Op intent } \\
\hline GTR & $310(69 \%)$ & $159(75 \%)$ \\
\hline Debulking & $90(20 \%)$ & $51(25 \%)$ \\
\hline Biopsy & $50(11 \%)$ & $0(0 \%)$ \\
\hline Prior treatment & $79(17.6 \%)$ & $43(20.5 \%)$ \\
\hline \multicolumn{3}{|l|}{ Radiological tumor factors } \\
\hline Potentially resectable & $324(72 \%)$ & $167(80 \%)$ \\
\hline \multicolumn{3}{|l|}{ Epicenter } \\
\hline Lobar & $365(82 \%)$ & $203(97 \%)$ \\
\hline Central & $33(7 \%)$ & $3(1 \%)$ \\
\hline Posterior fossa & $5(1 \%)$ & $1(1 \%)$ \\
\hline Other & $47(10 \%)$ & $3(1 \%)$ \\
\hline Multifocal & $12(3 \%)$ & $8(4 \%)$ \\
\hline \multicolumn{3}{|l|}{ Enhancement } \\
\hline Predominant & $209(46 \%)$ & $58(28 \%)$ \\
\hline Mixed & $112(25 \%)$ & $57(27 \%)$ \\
\hline None & $129(29 \%)$ & $95(45 \%)$ \\
\hline \multicolumn{3}{|l|}{ Tumor delineation } \\
\hline Good & $236(52 \%)$ & $97(46 \%)$ \\
\hline Moderate & $179(40 \%)$ & $93(44 \%)$ \\
\hline Poor & $35(8 \%)$ & $20(10 \%)$ \\
\hline \multicolumn{3}{|l|}{ Eloquent } \\
\hline Involved & $112(25 \%)$ & $52(25 \%)$ \\
\hline Close & $206(46 \%)$ & $104(50 \%)$ \\
\hline Not involved & $132(29 \%)$ & $54(25 \%)$ \\
\hline Histology: glial & $340(75 \%)^{*}$ & $210(100 \%)$ \\
\hline \multicolumn{3}{|l|}{ Op adjuncts used } \\
\hline $\begin{array}{l}\text { Intraop neurophysiological } \\
\text { monitoring used }\end{array}$ & $111(25 \%)$ & $69(33 \%)$ \\
\hline Awake craniotomy & $55(12 \%)$ & $33(16 \%)$ \\
\hline ALA-induced fluorescence & $44(10 \%)$ & $28(13 \%)$ \\
\hline
\end{tabular}

Values are presented as number (\%) of patients unless otherwise indicated. * These 340 gliomas comprise all of the gliomas operated using image guidance. Of these, only those gliomas for which US was used for resection control (as opposed to localization) and those having postoperative MRI for assessing the resection were included in the final analysis $(n=210)$.

US scan. In the remaining 113 cases (54\%), a total of 150 intermediate scans were performed (median 1, range 1-4 scans per patient). Intermediate scans were more likely to be performed for unresectable $(60 \%$ vs $52 \%$ for resectable), nonenhancing ( $60 \%$ vs $49 \%$ for enhancing), and noneloquent (61\% vs 52\% for eloquent) tumors and when US image quality was acceptable (54\% when good and 53\% when fair compared with $33 \%$ when it was poor). None of these differences were statistically significant. There was no difference in the likelihood of an intermediate scan be- 


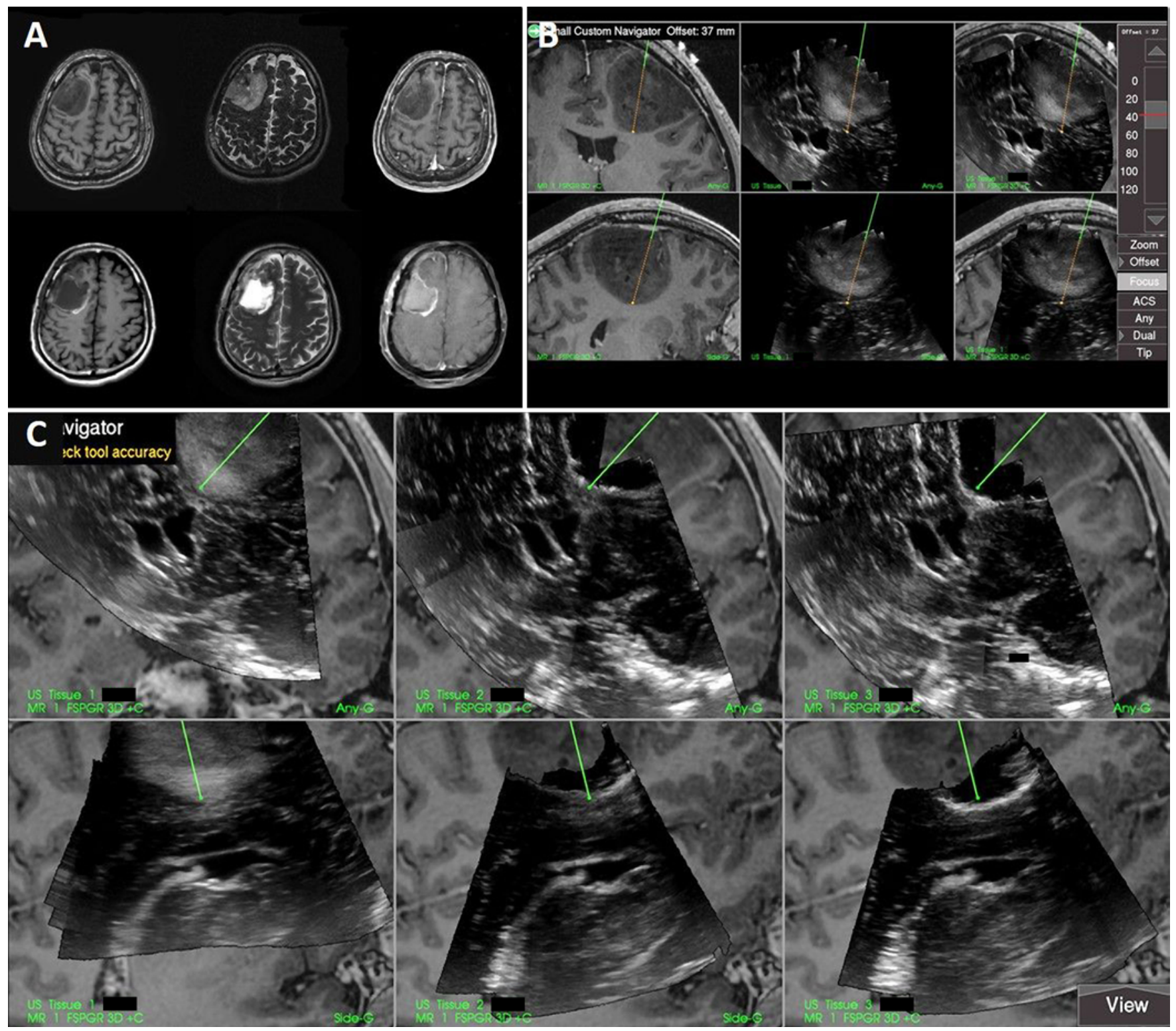

FIG. 2. A: The top row shows the preoperative MR images (left, T1; center, T2; right, postcontrast T1-weighted) and the bottom row shows postoperative MR images (left, T1; center, T2; right, postcontrast T1-weighted) of a right frontal nonenhancing glioma. The tumor was well circumscribed on MRI and completely excised. B: Intraoperative screenshots from the navigation system. GTR was achieved using NUS combined with preoperative MRI (image fusion). The left panels show the preoperative T1-weighted MR images depicted in two orthogonal planes (the top view is in the plane of the navigator and the bottom view is in a plane mutually perpendicular to that). The center panels show the volumetric US depicted in the same corresponding planes. The US image quality was good. The tumor is hyperechoic and well delineated. The focus of the US scan is set toward the bottom of the tumor so that the brain-tumor interface is well seen, as a result of which the superficial tumor is not imaged cleanly. The right panels show both US and MRI overlaid. The dotted line indicates a point just beyond the brain-tumor interface on the US image. Note that on the corresponding MR image, the same point seems to be on the edge of the tumor (representing a brainshift). Brainshift is also evident in the right panels (overlaid images) where the locations of the falx on MRI and US are not aligned. C: Serial resection control US scans of the same patient: preresection (left pane/s), intermediate (center panels), and postresection (right panels). All are depicted in the same dual orthogonal planes. Nodular, hyperechoic residual tumor can be seen in the intermediate scans (virtual navigator pointer). This residue was unanticipated and was subsequently resected. Complete resection was confirmed on the final US scan (linear thin, regular hyperechoic rim indicating no residual tumor). Histology was isocitrate dehydrogenase-mutant anaplastic astrocytoma. 

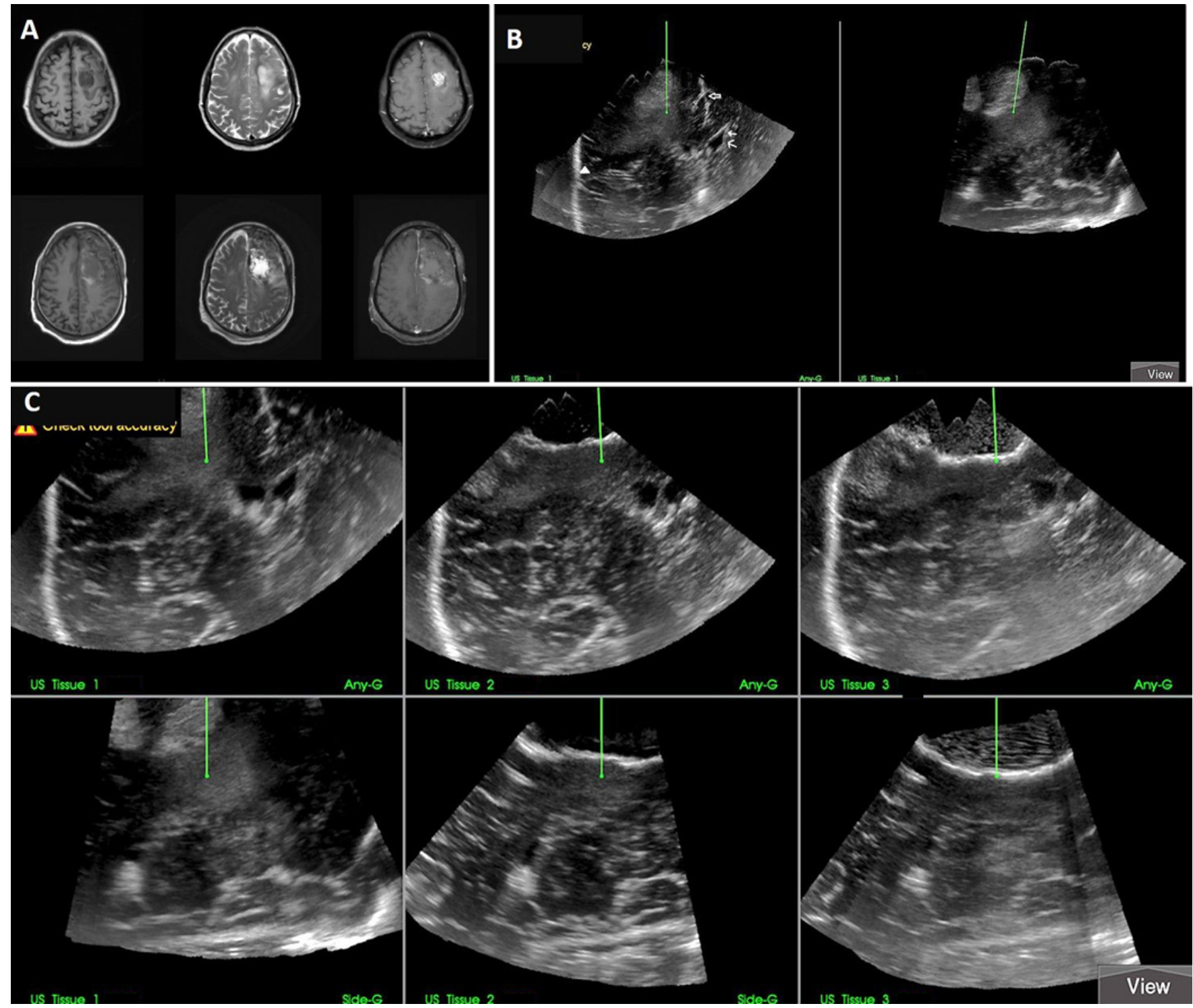

FIG. 3. Left frontal enhancing glioma. A: The top row shows the preoperative MR images (left, T1; center, T2; right, postcontrast T1-weighted). The tumor was moderately circumscribed on MRI. The bottom row shows the postoperative MR images (left, T1; center, T2; right, postcontrast T1-weighted). III-defined bright T2 signal intensities depict infiltrating residual tumor in the eloquent areas. B: Intraoperative screenshots from the navigation system. The patient underwent resection using DNUS (without preoperative MRI fusion imaging). Preresection 3D US images are depicted in orthogonal planes. The tumor appears to be variably hyperechoic, with less clearly defined margins. Anatomical structures seen include the falx (large arrow), frontal horn of the ventricle (line arrows), and bone of the frontal convexity (solid arrowhead). C: Serial resection US scans of same the patient as in panel B: preresection (left pane/s), intermediate (center pane/s), and postresection (right pane/s). The bright hyperechoic rim of the cavity is seen in the subsequent scans with a lesser hyperechoic residual tumor below it. This has been serially resected. Some residual hyperechoic tumor was left (STR) due to positive subcortical stimulation at the depths. Histology revealed isocitrate dehydrogenase-mutant glioblastoma.

ing done in HGGs and low-grade gliomas (LGGs) (54\% and 53\%, respectively). In 21 of the earliest cases (with 35 intermediate scans), data regarding the surgeon's interpretation of anticipated residue were not recorded. So intraoperative decision-making was evaluable in 115 intermediate scans in 92 patients. In 66 instances, the surgeon had anticipated a tumor residue that was confirmed in 64 patients going on to further resection, whereas in 2 patients no detectable tumor residue was seen on US, findings that led to stoppage of the resection. On the other hand, in 47 of the 49 times that intermediate scans were done with the assumption of a complete resection, unanticipated tumor residue was picked up by the US, prompting a further resection in all of these instances. Thus, the intermediate US scan altered the intraoperative decision in 49 (42.5\%) of the 115 times it was performed. 

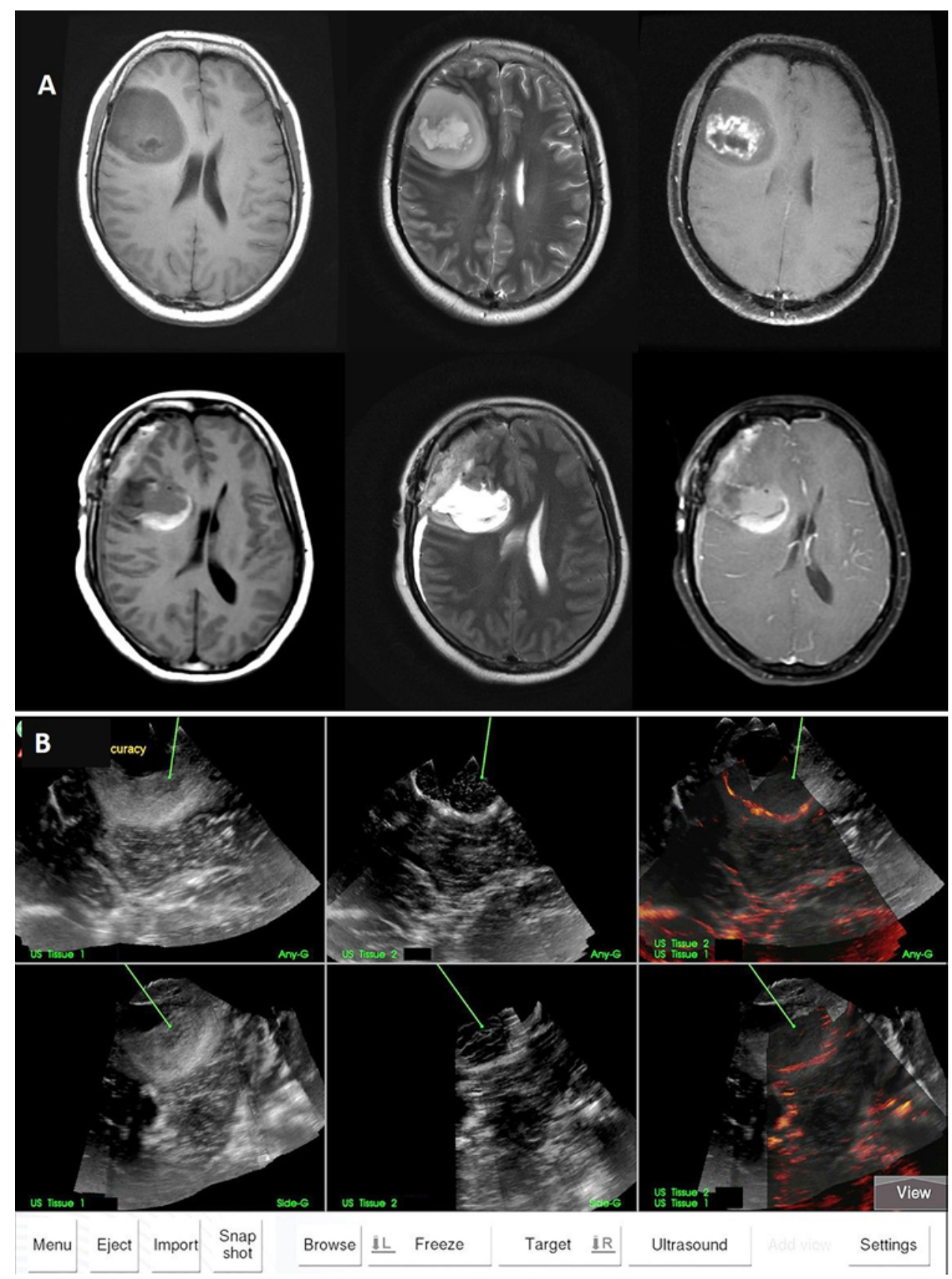

FIG. 4. A right frontal enhancing glioma. A: The top row shows the preoperative MR images (left, T1; center, T2; right, postcontrast T1-weighted). The tumor was well circumscribed on MRI. The bottom row shows the postoperative MR images (left, T1; center, T2; right, postcontrast T1-weighted). Postoperative MRI shows a rim of blood products along the cavity but no residual tumor. B: Intraoperative screenshots from the navigation system. The patient underwent resection using DNUS (without preoperative MRI fusion imaging). The tumor appears hyperechoic with well-defined margins on the preresection scan (left panel). In this case, no intermediate scan was performed. The final US scan (center panel) shows a clear hyperechoic cavity rim with no residual tumor. The right panel shows an overlay of the pre- and postresection US scans (the postresection scan has been shaded red to show the demarcation). Note the shift between the two sets of US images. The cavity seems to be slightly superficial compared with the deepest tumor margin, due to deformation. The absence of residual hyperechoic tissue beyond the cavity rim confirms complete resection. Histopathology was glioblastoma (isocitrate dehydrogenase wild type).

\section{EOR in Gliomas}

Eventually, US-defined GTR was obtained in $57.6 \%$ (121/210) of cases (55\% [66/120] and 61\% [55/90], respectively, for DNUS and image fusion US modes [p = $0.4]$ ). The US-defined GTR rate was higher in the group of patients in whom no intermediate US scans were performed than in the group in whom they were $(64 \%$ and $54 \%$, respectively, $\mathrm{p}=0.3$ ). Correspondingly, overall, the MR-defined GTR rate in this cohort of gliomas was $58.6 \%$ (123/210). In addition, near-total resection (NTR) (> 90\% 
TABLE 2. Diagnostic accuracy of resection control US in predicting the presence of residual tumor

\begin{tabular}{lcc}
\hline & \multicolumn{2}{c}{ Value } \\
\hline Final US: residue & Yes: 68 & No: 21 \\
\hline Final US: no residue & Yes: 19 & No: 102 \\
\hline Diagnostic accuracy statistics & & \\
\hline Sensitivity & $78.16 \%$ & $68.02-86.31 \%$ \\
\hline Specificity & $82.93 \%$ & $75.09-89.11 \%$ \\
\hline Positive likelihood ratio & 4.58 & $3.05-6.86$ \\
\hline Negative likelihood ratio & 0.26 & $0.18-0.40$ \\
\hline PPV & $76.40 \%$ & $68.35-82.92 \%$ \\
\hline NPV & $84.30 \%$ & $78.16-88.95 \%$ \\
\hline Overall accuracy & $80.95 \%$ & $74.98-86.03 \%$ \\
\hline
\end{tabular}

Values are presented as numbers or percentage or ratio and $95 \% \mathrm{Cl}$.

resection) was achieved in $18.6 \%(39 / 210)$, with the rest being subtotal resections (STRs) ( $<90 \%$ resection). GTR rates were lower in the gliomas for which an intermediate scan had been performed (55\% vs $61 \%, \mathrm{p}=0.21)$. Rates for both US- and MR-defined GTR were significantly higher in the intended GTR group than in the intended debulking group $(71.8 \%$ [114/159] vs $11.8 \%$ [6/51] and $71.8 \%$ [114/159] vs $17.6 \%$ [9/51], respectively). This difference was highly significant $(\mathrm{p}<0.0001$ for both US- and MRdefined GTR)

\section{Diagnostic Accuracy of Resection Control US in Gliomas ( $n=210$ )}

Overall concordance between US and MRI was seen in 170 of the gliomas (diagnostic accuracy of 81\%) (Table 2 ). The sensitivity and specificity of US in detecting tumor residue were $78 \%$ and $83 \%$, respectively. US missed detecting residual tumor in 19 cases (false negatives). Of these, NTR was achieved in 13 and STR in 6 cases. On the other hand, the final US scan had shown tumor residue in 21 cases (intentionally left because of proximity to eloquent areas) and postoperative MRI did not detect it (false positive).

\section{Factors Affecting GTR}

There was no difference in GTR rates between HGG and LGG $(60 \%$ vs $51 \%$, p = 0.83). Baseline preoperative variables like resectability, tumor delineation on MRI, eloquent location, and enhancement pattern, as well as the mode of US used and the iUS image quality, were evaluated for their influence on GTR rate. On univariate analysis, all of these factors except the US mode were significant (Table 3). On multivariate logistic regression analysis, only resectability and eloquent location remained significant (Table 4).

\section{Morbidity}

In the entire cohort, the overall morbidity was $18.5 \%$ (neurological worsening occurring in $13.5 \%, 7.8 \%$ being major). There was no difference in the morbidity rates in the glioma subgroup, with the corresponding values being similar (overall morbidity $21.4 \%$ and neurological worsening $16.8 \%$, with $6.7 \%$ being major).
TABLE 3. Univariate analysis of potential predictors of GTR

\begin{tabular}{lccc}
\hline & \multicolumn{2}{c}{ Extent of Resection } & \\
\cline { 2 - 3 } & GTR & Non-GTR & p Value \\
\hline Resectability & & & \\
\hline Resectable & $116(94.3)$ & $51(58.6)$ & $<0.0001$ \\
\hline Unresectable & $7(5.7)$ & $36(41.4)$ & \\
\hline MR delineation & & & \\
\hline Good & $75(61)$ & $22(25.3)$ & $<0.0001$ \\
\hline Moderate & $42(34.1)$ & $51(58.6)$ & \\
\hline Poor & $6(4.9)$ & $14(16.1)$ & \\
\hline Contrast enhancement pattern & & & \\
\hline Predominant & $43(35)$ & $15(17.2)$ & 0.011 \\
\hline Mixed & $27(22)$ & $30(34.5)$ & \\
\hline Negligible & $53(43.1)$ & $42(48.3)$ & \\
\hline US mode & & & \\
\hline DNUS & $76(61.8)$ & $54(62.1)$ & 0.701 \\
\hline Image fusion & $47(38.2)$ & $33(37.9)$ & \\
\hline US image quality & & & \\
\hline Good & $77(62.6)$ & $39(44.8)$ & 0.01 \\
\hline Moderate & $45(36.6)$ & $43(49.4)$ & \\
\hline Poor & $1(0.8)$ & $5(5.7)$ & \\
\hline Eloquent location & & & \\
\hline Noneloquent & $43(35)$ & $11(12.6)$ & $<0.0001$ \\
\hline Close & $58(47.2)$ & $46(52.9)$ & \\
\hline Eloquent involved & $22(17.9)$ & $30(34.5)$ & \\
\hline
\end{tabular}

Values are presented as number (\%) of patients unless otherwise indicated.

\section{Discussion}

We present the outcomes of using NUS in a large series of gliomas and specifically discuss the influence of NUS on the intraoperative decision-making process as well as the factors affecting the overall outcomes. Our results showed that NUS positively altered the decision-making process in a significant proportion of gliomas $(42.5 \%)$ in patients undergoing resective surgery using NUS. NUS predicted residual tumor with a relatively high diagnostic accuracy of $81 \%$ overall, achieving an overall GTR rate of $57 \%$ without additional morbidity. NUS assessment showed good concordance with postoperative MR assessment of GTR $(80 \%)$, though there were false negatives and positives. As a group, the eventual GTR rate was significantly influenced by the resectability and the eloquent location of the tumors.

In our overall experience, resection control was the main reason for using NUS (69\%). Considering only the subgroup of gliomas in our cohort, this is one of the largest such series of gliomas operated using NUS reported to date. Munkvold et al. reported a series of 144 gliomas operated using NUS. ${ }^{8}$ Their cohort of gliomas had a higher proportion of LGGs (33\% vs 17\%) and more recurrent cases (40\% vs 20\%) compared with our series. Munkvold et al. reported the diagnostic accuracy of NUS and the GTR rates but did not analyze the intraoperative decision-making based on NUS. 
TABLE 4. Multivariate logistic regression analysis of potential predictors of GTR

\begin{tabular}{lll}
\hline \multicolumn{1}{c}{ Variable } & OR $(95 \% \mathrm{Cl})$ & p Value \\
\hline Resectability & & \\
\hline Unresectable & & \\
\hline Resectable & $9.94(2.91-33.95)$ & $<0.001$ \\
\hline MR delineation & & \\
\hline Poor & $0.37(0.08-1.60)$ & 0.182 \\
\hline Fair & $0.73(0.14-3.77)$ & 0.711 \\
\hline Good & & \\
\hline Contrast enhancement pattern & & \\
\hline Negligible & $0.57(0.26-1.25)$ & 0.158 \\
\hline Mixed & $1.36(0.60-3.10)$ & 0.465 \\
\hline Predominant & & \\
\hline Eloquent & & \\
\hline Eloquent involved & $1.96(0.90-4.28)$ & 0.091 \\
\hline Close & $4.66(1.72-12.59)$ & 0.002 \\
\hline Noneloquent & & \\
\hline US image quality & & 0.903 \\
\hline Poor & $1.16(0.10-13.08)$ & \\
\hline Fair & $1.12(0.09-13.51)$ & 0.931 \\
\hline Good & & \\
\hline
\end{tabular}

\section{Intraoperative Decision-Making Based on NUS}

One of the benefits of using iUS is that rapid and frequent updates can be performed intraoperatively before a final resection control scan. Munkvold et al. ${ }^{8}$ had a high number of intermediate scans (mean of 3-7 depending on the image quality), whereas in our series, the number of intermediate scans was lower, reflecting subjectivity in the use of the serial US updates. It is likely (and this may be true for more experienced users) that the resection may have been adjudged complete on the first intermediate scan (which would then get counted as a final resection control scan and not an intermediate scan), or sometimes the surgeon may have determined that the intended debulking had been achieved (as in diffuse unresectable tumors where a GTR is not intended or in tumors close to eloquent regions where further resection may not be possible) and only a final-check US scan would be done for documentation. It is also possible that, as we often do, we performed a quick-check 2D US scan for visual confirmation, though a NUS scan may not have been performed and hence not documented. Whereas performing multiple intermediate scans is always recommended, the interpretation of the utility of each of such scans may be limited by the bias that can be associated with intentionally performing multiple check scans (as the surgeon would most probably be expecting a tumor residue and would go ahead with resection more often than not). On the other hand (as in our series), if fewer intermediate scans were performed, it could have been at times when the surgeon most needed the information from the updated US scan (with a lower propensity of a bias). Consequently, the intermediate scan altered the surgical decision in a large number of cases
( $42.5 \%$ of the times it was performed). Most of these were in fact unanticipated findings of tumor residues that were further resected and may have been missed if not for the imaging. This result underlines a very crucial benefit of the intraoperative imaging. It has been shown that without intraoperative resection control, surgeons tend to overestimate GTR in up to $70 \%$ cases that are potentially resectable. ${ }^{9}$ A major cause of this is inadvertently missed residues, and these are usually small remnants toward the end of the glioma resection, accounting for a lower than expected GTR rate ( $24 \%$ in that series) in a potentially resectable cohort of gliomas. In our series, the use of NUS for resection control led to an eventual US-defined GTR in around $57 \%$ of cases (the GTR rate was $70 \%$ in the resectable group) (Table 3). Interestingly, the GTR rate was higher in the subgroup where intermediate scans were not performed (64\% vs 54\%). This result corroborates our hypothesis that in cases where intermediate scans were not performed, the surgeon was more confident of the resection, which may have been a function of the better resectability of these tumors, which, as we have shown, was an independent predictor of GTR by itself.

\section{Concordance Between Resection Control US and MRI and Diagnostic Accuracy of NUS}

The overall GTR rate was $58 \%$. This was higher than the $27 \%$ rate reported by Munkvold et al. ${ }^{8}$ This apparent difference could be because Munkvold et al. used a stricter volumetric assessment of MRI, which we did not (though this would not have significantly affected the definition of "no residue" as a dichotomized variable). Additionally, our cohort had a larger proportion of HGGs, for which the MRI definition of residue based on T1-contrast is easier, than LGGs, which may be more diffuse, and therefore estimation of residual tumor based on T2/FLAIR MRI may be more challenging. Although US GTR and MRI GTR rates were similar (57\% and 58\%) in our study, it is important to note that the intermodality concordance was $81 \%$. This implies that merely comparing the GTR rates of the two modalities can be misleading, as the overall rates may appear identical and yet mask false positives and negatives. One must also raise the possibility that the MRI may be erroneous in the immediate postoperative setting, and using it as a gold standard for assessing the diagnostic accuracy of other modalities (e.g., US) may be unreliable. ${ }^{10}$ Indeed, a study comparing coregistered intraoperative MRI, iUS, and aminolevulinic acid (ALA) in glioblastomas undergoing resection showed that concordance (using histopathology as the gold standard) was higher with ALA and iUS compared with intraoperative MRI. ${ }^{11}$ Nonetheless, postoperative MRI remains the standard of care to assess the EOR, and NUS compares favorably to the MRI (if not better). In our study, the specificity (i.e., the ability to correctly rule out residue in all cases where no residue exists) and positive predictive value (PPV) (the likelihood of a US-predicted residue truly being residual disease) were high ( $83 \%$ and $76 \%$, respectively), though slightly lower than those reported by Munkvold et al. (85\% and $89 \%$, respectively). ${ }^{8}$ Additionally, the sensitivity (the ability to correctly rule in a residual tumor in all cases with residual tumor) and negative predictive value (NPV) (the likelihood of a US-predicted "no residue" truly 
being the absence of residue) were also high (78\% and $84 \%$, respectively) compared with the much lower rates reported by Munkvold et al. (46\% and 37\%, respectively), leading to a better overall diagnostic accuracy. This improved sensitivity, with a lower false-negative rate $(22 \%)$ in our series, could have contributed to the higher GTR rate. Even with higher false negatives as reported by Munkvold et al., the residual tumor volumes were quite small (mean $<1 \mathrm{ml}$ ). Similarly, in our study, of the 19 false-negative cases, 13 were NTRs and the remaining 6 were STRs. This means that even though there can be false negatives with NUS, it is unlikely to miss large residues and the overall resection rates will be significantly higher. This was also emphasized in a larger meta-analysis. ${ }^{10}$ The overall diagnostic accuracy of NUS in our hands was $81 \%$, which compares favorably to contemporary published series ${ }^{8}$ as well as a meta-analysis ${ }^{10}$ assessing the diagnostic accuracy of iUS. Interpretation of the resection control scan remains the key in improving the predictive value of US. All attempts should be made to obtain optimal scan quality by minimizing artifacts., ${ }^{5,12}$ User experience is also very important, and user subjectivity in interpretation can contribute to the variable diagnostic accuracies reported across studies. ${ }^{10}$

\section{Factors Influencing Resection Rates}

We also evaluated various tumor-related factors that influenced the resection rates. Not surprisingly, tumor resectability and eloquent location were the two main determinants. It is worthwhile noting here that though this was a selected cohort of gliomas planned for radical surgery, not all were deemed resectable (resectability was $80 \%$ ) (Table 1). Even though the GTR rates were significantly better in the resectable group (70\%), it is important to note that even in the so-called unresectable group, $17 \%$ had a GTR. This reinforces the fact that a radical intent yields superior results in terms of resection rates. ${ }^{13}$ It also highlights the challenges in defining "resectability," which is actually a very subjective and arbitrary definition..$^{14}$ It is a composite indicator of many variables (including, but not limited to, tumor size, delineation, location, eloquence, and pattern of contrast enhancement). Whereas some of these are quantifiable, others are not. Smaller size and superficial location (both surrogate markers of " resectable" tumors) have been shown to be significant predictors of GTR when using NUS for gliomas. ${ }^{8}$ Eloquence itself, as defined by preoperative evaluation, is also a relative definition as has been demonstrated by Chang et al., putting forth the concept of "presumed eloquence," which can be verified (and modified) only by intraoperative mapping. ${ }^{15}$ Extrapolating the concept to resectability, an optimal strategy would be to consider all gliomas eligible for debulking surgery (excluding those undergoing biopsy) to be presumably resectable and offer the best surgical outcomes using a multimodal approach (with intraoperative image guidance like NUS, in our experience). As evident in our study, even in so-called unresectable tumors, using NUS with the intent to attempt radical resection, up to $17 \%$ of patients may benefit from a GTR.

\section{Limitations}

This was a retrospective study and hence subject to the limitations of such a study. Some of the data especially related to postoperative MR assessments were incomplete and excluded from the analysis. This was also a highly selected cohort of patients comprising lobar tumors (predominantly gliomas) that were candidates for radical surgery, which could have been influenced by the distillation of our experience and preferred choice over a period of time, leading to a selection bias. Our findings also cannot be used to differentiate the potential benefits and limitations of NUS versus standard 2D US as the study lacked a comparative arm. Though 2D US is part of the system that can perform NUS, it was rarely used as such, especially for this cohort of gliomas. Nevertheless, within this selected group of lobar glioma cases, we were able to explore the role of NUS in intraoperative decision-making and evaluate its influence on the surgical outcomes.

\section{Conclusions}

NUS is a useful intraoperative adjunct for resection control in gliomas, detecting unanticipated tumor residues and positively influencing the course of the resection, leading to higher resection rates. EOR is determined by the innate resectability of the tumor and its relationship to eloquent locations, reinforcing the need to combine iUS with functional mapping techniques to optimize resections.

\section{Acknowledgments}

The authors would like to thank Robin John and Deepak Prajapati for their help in the data collection and Akshay Patil for helping with the statistical analysis.

\section{References}

1. Moiyadi A, Shetty P. Objective assessment of utility of intraoperative ultrasound in resection of central nervous system tumors: a cost-effective tool for intraoperative navigation in neurosurgery. J Neurosci Rural Pract. 2011;2(1):4-11.

2. Moiyadi A. Intraoperative ultrasound in neurosurgical oncology-scope and utility. In: Hayat MA, ed. Tumors of the Central Nervous System. Vol 13. Springer Netherlands; 2014: 149-158.

3. Moiyadi AV, Shetty P. Direct navigated 3D ultrasound for resection of brain tumors: a useful tool for intraoperative image guidance. Neurosurg Focus. 2016;40(3):E5.

4. Patil AD, Singh V, Sukumar V, et al. Comparison of outcomes of free-hand 2-dimensional ultrasound-guided versus navigated 3-dimensional ultrasound-guided biopsy for supratentorial tumours: a single-institution experience with 125 cases. Ultrasonography. 2019;38(3):255-263.

5. Moiyadi AV, Shetty PM, Mahajan A, et al. Usefulness of three-dimensional navigable intraoperative ultrasound in resection of brain tumors with a special emphasis on malignant gliomas. Acta Neurochir (Wien). 2013;155(12):2217-2225.

6. Moiyadi AV, Shetty P, John R. Non-enhancing gliomas: does intraoperative ultrasonography improve resections? Ultrasonography. 2019;38(2):156-165.

7. Moiyadi AV, Kannan S, Shetty P. Navigated intraoperative ultrasound for resection of gliomas: predictive value, influence on resection and survival. Neurol India. 2015;63(5):727-735.

8. Munkvold BKR, Jakola AS, Reinertsen I, et al. The diagnostic properties of intraoperative ultrasound in glioma surgery and factors associated with gross total tumor resection. World Neurosurg. 2018;115:e129-e136.

9. Orringer D, Lau D, Khatri S, et al. Extent of resection in 
patients with glioblastoma: limiting factors, perception of resectability, and effect on survival. J Neurosurg. 2012;117(5): 851-859.

10. Trevisi G, Barbone P, Treglia G, et al. Reliability of intraoperative ultrasound in detecting tumor residual after brain diffuse glioma surgery: a systematic review and meta-analysis. Neurosurg Rev. 2020;43(5):1221-1233.

11. Coburger J, Scheuerle A, Pala A, et al. Histopathological insights on imaging results of intraoperative magnetic resonance imaging, 5-aminolevulinic acid, and intraoperative ultrasound in glioblastoma surgery. Neurosurgery. 2017;81(1): 165-174.

12. Selbekk T, Jakola AS, Solheim O, et al. Ultrasound imaging in neurosurgery: approaches to minimize surgically induced image artefacts for improved resection control. Acta Neurochir (Wien). 2013;155(6):973-980.

13. Solheim O, Selbekk T, Jakola AS, Unsgård G. Ultrasoundguided operations in unselected high-grade gliomas - overall results, impact of image quality and patient selection. Acta Neurochir (Wien). 2010;152(11):1873-1886.

14. Sonabend AM, Zacharia BE, Cloney MB, et al. Defining glioblastoma resectability through the wisdom of the crowd: a proof-of-principle study. Neurosurgery. 2017;80(4):590_ 601.

15. Chang EF, Clark A, Smith JS, et al. Functional mappingguided resection of low-grade gliomas in eloquent areas of the brain: improvement of long-term survival. J Neurosurg. 2011;114(3):566-573.

\section{Disclosures}

The authors report no conflict of interest concerning the materials or methods used in this study or the findings specified in this paper.

\section{Author Contributions}

Conception and design: Moiyadi, Shetty. Acquisition of data: all authors. Analysis and interpretation of data: all authors. Drafting the article: Moiyadi, Shetty. Critically revising the article: all authors. Reviewed submitted version of manuscript: all authors. Approved the final version of the manuscript on behalf of all authors: Moiyadi. Statistical analysis: Moiyadi, Shetty. Administrative/technical/material support: Moiyadi. Study supervision: Moiyadi, Shetty.

\section{Supplemental Information \\ Online-Only Content}

Supplemental material is available online.

Supplementary Fig. 1. https://thejns.org/doi/suppl/10.3171/ 2020.10.FOCUS20550.

\section{Correspondence}

Aliasgar Moiyadi: Tata Memorial Centre, Mumbai, India. aliasgar. moiyadi@gmail.com. 